\title{
AERAÇÃO NASAL EM CRIANÇAS ASMÁTICAS
}

\section{Nasal ventilation in asthmatics children}

\author{
Daniele Andrade da Cunha ${ }^{(1)}$, Hilton Justino da Silva ${ }^{(2)}$, Klyvia Juliana Rocha de Moraes ${ }^{(3)}$, \\ Renata Andrade da Cunha ${ }^{(4)}$, Renata Milena Freire Lima Régis ${ }^{(5)}$, Elthon Gomes Fernandes da Silva ${ }^{(6)}$, \\ Gerlane Karla Bezerra Oliveira Nascimento ${ }^{(7)}$, Gutemberg Moura de Andrade ${ }^{(8)}$, \\ Silvia Regina Arruda de Moraes ${ }^{(9)}$, Célia Maria Machado Barbosa de Castro ${ }^{(10)}$
}

\begin{abstract}
RESUMO
Objetivo: identificar a presença de sinais de alterações na expiração de crianças asmáticas. Método: este estudo foi realizado com 30 crianças com idades entre 6 e 10 anos de ambos os sexos com diagnóstico clínico de asma confirmado pelo prontuário médico e 30 crianças não asmáticas também de ambos os sexos na mesma faixa etária. Foi avaliada a aeração nasal com o espelho milimetrado de Altmann, sendo mensurado o escape de ar nasal objetivando a verificação da saída uni ou bilateral do ar e a relação de simetria entre a narina direita e a esquerda. As imagens foram importadas para o computador por meio do scanner HP da série Scanjet 2400. A análise foi realizada no software Scion Image for Windows (Alpha 4.0.3.2). Para análise das variáveis quantitativas entre grupos foi aplicado o teste t-student e para a análise intragrupos foi aplicado o teste t-Student pareado.Todas as conclusões foram tomadas ao nível de significância de 5\%, sendo usados o Excel 2000 e o SPSS v8.0, para as análises. Resultados: não foram encontradas diferenças estatisticamente significantes entre as crianças asmáticas e não-ásmáticas, acerca das mensurações quanto à área total e quanto às áreas das narinas direita e esquerda. Conclusão: não foi identificada a presença de sinais de alterações na expiração de crianças asmáticas, desta forma, faz-se necessário um estudo mais específico das funções nasal e pulmonar.
\end{abstract}

DESCRITORES: Asma; Criança; Obstrução Nasal; Expiração

(1) Fonoaudióloga, Pesquisadora Institucional da Faculdade Integrada do Recife - Estácio FIR, Recife-PE, Brasil; Doutora em Nutrição pela Universidade Federal de Pernambuco.

(2) Fonoaudiólogo, Vice-Coordenador do Programa de PósGraduação em Patologia, nível Mestrado, Docente do Curso de Fonoaudiologia da Universidade Federal de Pernambuco, UFPE, Recife-PE, Brasil; Doutor em Nutrição pela Universidade Federal de Pernambuco.

(3) Fisioterapeuta, Preceptora de estágio em Fisioterapia Cárdio-Pulmonar pela Universidade Salgado de Oliveira, Mestranda em Patologia pela Universidade Federal de Pernambuco, UFPE, Recife-PE, Brasil; Especialista em Fisioterapia Cárdio-Respiratória pela Universidade Gama Filho

(4) Fisioterapeuta; Especialista em Fisioterapia Neurofuncional pela Faculdade Integrada do Recife

(5) Fonoaudióloga; Especialista em Motricidade Orofacial pela Faculdade Integrada do Recife.

(6) Fonoaudiólogo, Mestrando em Saúde Humana e Meio Ambiente pela Universidade Federal de Pernambuco, UFPE, Recife-PE

(7) Fonoaudióloga, Mestranda em Patologia Universidade Federal de Pernambuco, UFPE, Recife-PE

\section{INTRODUÇÃO}

A asma brônquica, em particular na infância, é um transtorno crônico inflamatório das vias aéreas inferiores, caracterizado por obstrução ao fluxo de ar, na maioria das vezes de forma variável e reversível espontaneamente ou com o tratamento ${ }^{1,2}$.

(8) Fonoaudiólogo, Mestrando em Ciências da Computação, Universidade Federal de Pernambuco, UFPE, Recife-PE

(9) Fisioterapeuta; Doutora em Neurociências pela Universidade de São Paulo - SP; Professor adjunto da Universidade Federal de Pernambuco, Recife - PE, Brasil

(10) Médica; Doutora em Farmacologia pela Universidade Federal de Pernambuco; Professor Associado da Universidade Federal de Pernambuco, Recife - PE, Brasil

Justificativa pelo número de autores exceder 8: Este artigo é parte da tese de doutorado da Msc. Daniele Cunha, submetida à Universidade Federal de Pernambuco e todos os autores aqui citados participaram da coleta e análise dos dados da pesquisa. Conflito de interesses: inexistente 
A reação inflamatória da mucosa das vias aéreas inferiores leva às características clínicas da doença, como episódios recorrentes de tosse produtiva, broncoespasmo, edema da mucosa das vias aéreas inferiores, constricção torácica, dispnéia e sibilância ${ }^{3,4,5,6}$. Durante a crise asmática, o espasmo, o edema e a hipersecreção são fatores responsáveis pela obstrução brônquica, com prejuízo das duas fases da respiração tornando a inspiração rápida e superficial e a expiração ineficaz, gerando a hiperinsuflação pulmonar ${ }^{3}$. Os sintomas ocorrem predominantemente à noite, após exercícios físicos, mudanças bruscas de temperatura ou contato com substâncias inaladas (fumaça de cigarro, cigarro, perfumes, amônia, cloro, agentes de limpeza e outras substâncias químicas com odores fortes ${ }^{5}$ ) que funcionam como irritantes à via aérea ${ }^{5,6}$.

A partir da intensidade e frequência dos sintomas e da função pulmonar, a asma poderá ser classificada de acordo com sua gravidade como intermitente, persistente leve, moderada e grave ${ }^{5}$. Desta forma, os tratamentos são focados no quadro sintomático das crises e na profilaxia, propondo-se a minimizar sintomas e evitar novas exacerbações agudas, sendo utilizados para isto os broncodilatadores e antiinflamatórios ${ }^{4}$.

Em estudo com uma amostra randômica de pacientes que participaram do European Community Respiratory Health Survey (ERHS), foi verificado que a asma estava fortemente associada com a rinite, mesmo em pacientes não atópicos ${ }^{7}$.

A criança asmática que apresenta dificuldade respiratória e redução do fluxo aéreo nasal devido a uma obstrução de via aérea superior tem probabilidade maior de respirar pela boca, o que faz com que os músculos abaixadores da mandíbula efetuem sobre a mesma uma tração para trás a cada inspiração ${ }^{8}$.

Sendo assim, há várias formas de avaliação da função nasal, sendo que a mais conhecida e talvez uma das mais antigas seja o espelho milimetrado de Altmann ${ }^{9}$. Ele é frequentemente empregado para incentivar o uso funcional do nariz e mensurar a aeração nasal ${ }^{10,11}$, medindo $\circ$ ar expirado em pacientes com obstrução em vias aéreas superiores $^{10}$. Diversos estudos trazem o emprego do espelho milimetrado de Altmann como forma de comparação do fluxo aéreo nasal do mesmo indivíduo antes e após alguma intervenção $0^{9,12}$.

A maior parte da literatura traz consigo as alterações de vias aéreas inferiores que ocorrem em um indivíduo asmático, porém, ainda há uma escassez de estudos a respeito do comportamento aéreo nasal em crianças asmáticas. Sendo assim, o objetivo deste trabalho foi identificar a presença de sinais de alterações na expiração de crianças asmáticas.

\section{MÉTODO}

Trata-se de um estudo observacional, descritivo e transversal, o qual foi realizado no Ambulatório de Alergologia do Hospital das Clínicas da Universidade Federal de Pernambuco (UFPE) durante a primeira quinzena do mês de Abril/2008.

O grupo de estudo foi composto por 30 crianças com idades entre 6 e 10 anos, de ambos os sexos, e diagnóstico clínico de asma confirmado por meio de prontuário médico. Também foi avaliado um grupo controle com 30 crianças não-asmáticas, de faixa etária entre 6 e 10 anos, de ambos os sexos, mediante diagnóstico médico comprobatório da ausência da patologia.

Em ambos os grupos de estudo foram excluídas da pesquisa crianças com: comprometimento neurológico; crise de asma no momento da avaliação; portadores de cardiopatias graves; aparelho ortodôntico no momento da avaliação; anormalidades craniofaciais presentes, hipertrofias de amídalas e/ou adenóides e respiração oral. Todos os critérios de inclusão e exclusão foram obtidos através da análise individual e criteriosa de prontuários e também de contato direto com o médico responsável pelo voluntário, solicitando que o mesmo encaminhasse para a coleta apenas as crianças que atendessem aos critérios de estabelecidos nesta pesquisa.

Para a avaliação da aeração nasal foi utilizado espelho milimetrado de Altmann de acordo com as instruções de uso do produto. Este espelho consta em uma placa metálica, com um lado liso e outro com marcação milimetrada. O espelho foi colocado logo abaixo do nariz do voluntário que se encontrava sentado e com a cabeça reta durante a avaliação. Após duas expirações foi mensurado o escape de ar nasal, marcando com hidrocor azul a área embaçada, ao passo que, foi utilizada para anotação uma folha especial, milimetrada como o espelho. A análise da aeração nasal objetivou a verificação da saída uni ou bilateral do ar e a relação de simetria entre a narina direita e a esquerda. Os dados obtidos da coleta foram analisados por fonoaudiólogos e fisioterapeutas, por meio da observação dos esboços de aeração nasal (Figura 1).

Os responsáveis pelas crianças permitiram a participação destas na pesquisa por meio da assinatura de um Termo de consentimento livre e esclarecido. Não foram evidenciados riscos às crianças avaliadas na prática dessa pesquisa, contudo houve a possibilidade de algum desconforto emocional pelo fato de estarem sendo avaliadas. A pesquisa 


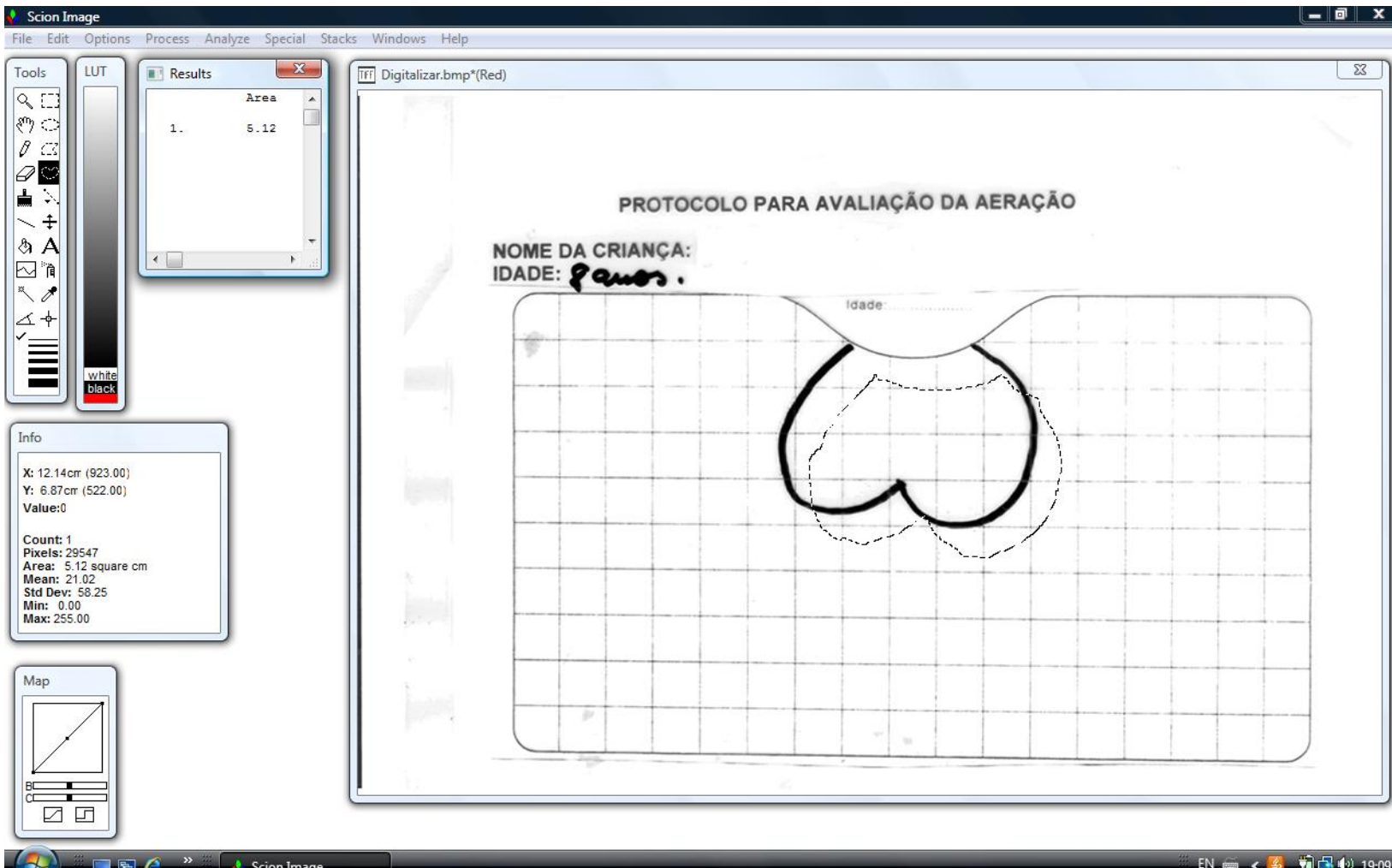

Figura 1 - Esboço da aeração nasal, em que o traçado contínuo representa a aferição da aeração nasal do paciente e o traçado pontilhado representa a mensuração pelo software Sçion Image para determinação da área.

foi aprovada pelo Comitê de Ética em pesquisas com seres humanos da UFPE sob o protocolo de n- 224/2006.

Após cada avaliação, o espelho de Altmann passou por um processo de assepsia utilizando algodão com uso do produto Germi-rio, que evita o acúmulo de germes patogênicos no espelho. Só após este processo, realizou-se uma nova avaliação. As imagens foram importadas para o computador por meio do scanner HP da série Scanjet 2400. A análise foi realizada no software Scion Image for Windows (Alpha 4.0.3.2). Neste programa foi utilizada a mensuração da área de acordo com a transformação da escala de 76 pixels por $1 \mathrm{~cm}^{2}$.

Foi realizada uma análise descritiva para expor os resultados obtidos. A apresentação das variáveis mensuradas foi feita por meio de tabelas, incluindo também o uso de algumas medidas descritivas como mínimo, máximo, média, desvio padrão e coeficiente de variação. Para testar a suposição de normalidade das variáveis envolvidas no estudo foi aplicado o teste de Kolmogorov-Smirnov. Para análise das variáveis quantitativas entre grupos foi aplicado o teste t-student. Para a análise intragrupos foram aplicados o teste t-Student pareado.
Sendo todas as conclusões tomadas ao nível de significância de 5\%. Os software utilizados para a análise foram o Excel 2000 e o SPSS v8.0.

\section{RESULTADOS}

Os resultados encontrados demonstraram a aeração nasal por meio da área total das narinas bem como da área de cada narina, tanto na população asmática quanto na população controle, expressos $\mathrm{em} \mathrm{cm}^{2}$ (por meio de valor mínimo, valor máximo, média e desvio padrão) após a conversão de 76 pixels por $1 \mathrm{~cm}^{2}$, por meio do software Scion Image for Windows (Alpha 4.0.3.2).

A tabela 1 apresenta os dados das mensurações do nível de aeração nasal entre as crianças asmáticas e não-asmáticas quanto à área total das narinas $\left(\mathrm{em} \mathrm{cm}^{2}\right)$. Nesta, não verificamos diferenças significantes.

A tabela 2 apresenta os dados da análise da aeração nasal quanto às áreas das narinas direita e esquerda $\left(\mathrm{em} \mathrm{cm}^{2}\right)$ nas crianças asmáticas e não-asmáticas. E nesta não verificamos diferenças significantes. 
Tabela 1 - Níveis de aeração nasal quanto à área total das narinas e comparação entre as crianças asmáticas e não-asmáticas

\begin{tabular}{lcccccc}
\hline Área Total $\left(\mathbf{c m}^{2}\right)$ & N & Mínimo & Máximo & Média & $\begin{array}{c}\text { Desvio- } \\
\text { padrão }\end{array}$ & p-valor \\
\hline Controle & 30 & 6,78 & 34,04 & 16,35 & 5,34 & \\
Asmático & 30 & 10,41 & 36,14 & 18,18 & 5,80 & 0,208 \\
\hline
\end{tabular}

Tabela 2 - Níveis de aeração nasal quanto às áreas das narinas direitas e esquerdas e comparação entre as crianças asmáticas e não-asmáticas

\begin{tabular}{lcccccc}
\hline Área Narina $\left(\mathbf{c m}^{2}\right)$ & $\mathbf{N}$ & Mínimo & Máximo & Média & $\begin{array}{c}\text { Desvio- } \\
\text { padrão }\end{array}$ & p-valor \\
\hline Controle & & & & & & \\
Direita & 30 & 3,58 & 15,95 & 8,55 & 2,76 & \\
Esquerda & 30 & 3,32 & 18,02 & 7,93 & 2,91 & 0,070 \\
\hline Asmáticos & & & & & & \\
Direita & 30 & 4,31 & 18,66 & 9,04 & 2,97 & \\
Esquerda & 30 & 3,09 & 17,52 & 9,15 & 3,36 & 0,806 \\
\hline
\end{tabular}

\section{DISCUSSÃO}

Os estudos mostram que há uma inter-relação entre a via aérea superior e a via aérea inferior, tanto em indivíduos sadios, quanto se comparados a uma população asmática e/ou rinítica ${ }^{1,7}$. Desta forma, há uma semelhança entre a constituição tecidual e arquitetônica destas vias aéreas, visto que a mucosa nasal e brônquica tem em sua formação o epitélio pseudo-estratificado, com células ciliadas colunares, apoiadas sobre a membrana basal ${ }^{6}$.

Um estudo avaliando pacientes não-asmáticos e com sintomas respiratórios, realizando a broncoprovocação com histamina monitorada com a curva fluxo-volume, verificou que houve a presença de hiper-responsividade brônquica em $52 \%$ dos casos, hiper-responsividade de vias extratorácicas (vias aéreas superiores) em $71 \%$, e de modo simultâneo em $41 \%$ apesar destes indivíduos não serem asmáticos, demonstrando a interdependência das vias aéreas como um todo ${ }^{13}$.

Desta forma, os achados da presente pesquisa, de acordo com a tabela 1, mostram para a área total das narinas, maiores valores de aeração nasal para as crianças asmáticas quando se comparado às crianças sem comprometimento asmático (grupo controle). Estes achados podem ser justificados, de acordo com a literatura, pelo fato de o asmático fazer uso prolongado de esquemas de drogas que são preventivas, destinadas a evitar o aparecimento das crises asmáticas por meio do combate à inflamação dos brônquios e das vias aéreas superiores (corticosteróides), assim como a utilização de medicamentos que irão agir direcionalmente à sintomatologia da doença, os chamados broncodilatadores ${ }^{4}$.

Os estudos postulam ainda que estes broncodilatadores podem ser de ação rápida a fim de prevenir os sintomas súbitos da asma, ou de ação lenta que servirão para prevenir por mais tempo o estreitamento da via aérea como um todo, evitando que isto aconteça ${ }^{4,14}$.

Todos estes estudos acima citados, que referem à interdependência nas vias aéreas (superiores e inferiores), bem como a ação dos esquemas medicamentosos, justificam os achados da tabela 1. Associado a este aspecto tem-se a possibilidade de ainda não haver a presença de limitações estruturais instauradas nestes asmáticos. É importante lembrar que na maioria das vezes, as limitações ao fluxo aéreo são variáveis e potencialmente reversíveis espontaneamente ou com o tratamento ${ }^{1,2,6}$.

O estudo de Degan e Puppin-Rontani ${ }^{15}$, avaliou o modo respiratório com o Espelho nasal milimetrado de Altmann. A aeração nasal foi avaliada em 20 crianças com hábitos de sucção como chupeta e mamadeira, numa faixa etária de 4 a 5 anos. Os autores verificaram que houve uma relação direta 
entre hábitos de sucção e aeração nasal, ou seja, as crianças utilizando chupeta e/ou mamadeira, apresentavam aeração nasal mais prejudicada; ao passo que afastando estes fatores, sua aeração nasal aumentava significantemente.

Os dados da tabela 2 demonstram que a média dos valores referentes às narinas direita e esquerda do grupo controle tem maiores discrepâncias e menores valores, quando comparados ao grupo asmático, respectivamente, ou seja, foi verificada uma maior assimetria (devido a essa discrepância em média) para a aeração no grupo controle. Estes achados entram em desacordo com os estudos de Branco, Ferrari e Weber ${ }^{16}$, que referem à asma como uma doença inflamatória causadora de obstrução nasal ao fluxo de ar gerando desta forma, uma redução do fluxo aéreo, sendo este fluxo de forma irregular.

No estudo que analisou a respiração oral e suas repercussões no estado nutricional de crianças, foram avaliadas 67 crianças com respiração oral, secundária à rinite alérgica e 134 crianças com respiração nasal para o grupo controle, numa faixa etária de 6 a 10 anos, observou-se saída de ar de forma bilateral tanto para os respiradores nasais (100\% dos casos) quanto para os respiradores orais $(83,1 \% \text { dos casos })^{17}$. Estes achados contribuem com a pesquisa atual, pois foi verificada a aeração nasal em $100 \%$ da amostra. Porém, no estudo de Cunha et al. ${ }^{17}$, não foi avaliada a aeração nasal para cada narina, como mostrada na tabela 2, o que pode gerar limitações no que diz respeito à aeração segmentada para cada narina, nas duas populações estudadas.

Pode-se ainda justificar a assimetria encontrada no grupo controle, pelo fato do diagnóstico da asma ainda ser um tanto quanto conflituoso. Os estudos mostram que a maioria das crianças que apresentam episódios recorrentes de sibilância após os 5 anos de idade é asmática, no entanto, o diagnóstico de asma antes dessa idade ainda é um problema e de certa forma, difícil ${ }^{18,19}$. A literatura também aponta para o diagnóstico neste período de vida, como sendo eminentemente clínico, não existindo meios propedêuticos disponíveis, rotineiramente, que permitam esclarecê-los com certeza ${ }^{20}$. Sendo assim, muitas das crianças avaliadas e triadas para o grupo controle, podem ter apresentado episódios de sibilância e cansaço, sem apresentarem diagnóstico prévio para asma, nem tão pouco fazerem uso de esquemas de drogas broncodilatadoras $e$ corticosteróides.
Além do que, há uma dificuldade de padronização para os protocolos relacionados à avaliação da aeração nasal11,17,21, podendo esta ser avaliada por meio do espelho milimetrado a partir da ausência ou presença do embaçamento ${ }^{11,21}$, quanto aos espaços marcados no próprio espelho ${ }^{15}$, ou do programa de computador ${ }^{9,22}$ resultando numa possível interferência nos resultados encontrados ${ }^{17}$. Mesmo assim, a avaliação da aeração nasal pelo método do espelho milimetrado, ainda é a forma mais utilizada pela facilidade de manuseio e baixo custo, porém tem certo grau de subjetividade, por medir apenas o ar expirado enquanto que os pacientes que apresentam obstrução de vias aéreas superiores mostram-se com dificuldades principalmente da fase inspiratória do ciclo respiratório ${ }^{23}$.

A literatura cita a Rinomanometria Computadorizada e a Rinometria acústica, como sendo métodos mais específicos e objetivos para a avaliação desta função nasal24, porém há uma dependência de equipamentos caros, o que justifica sua não utilização de rotina ${ }^{23}$. Alguns autores ainda relatam que as desordens das vias aéreas superiores podem resultar em desajustes na dinâmica respiratória e há também um aumento da resistência nasal, pois os músculos acessórios da inspiração passam a ser solicitados, o que leva a um movimento inspiratório proeminente do tórax superior ${ }^{25}$.

O presente estudo sinaliza a necessidade de novas pesquisas aprofundadas, que contribuam com este, possibilitando um melhor esclarecimento acerca da aeração nasal em crianças asmáticas, já que os dados da literatura a respeito de seu comprometimento e função ainda são escassos, o que dificulta a padronização da avaliação dos dados da aeração nasal, interferindo diretamente na abordagem desta população e em sua qualidade de vida.

\section{CONCLUSÃO}

Não foi identificada a presença de sinais de alterações na expiração de crianças asmáticas.

\section{AGRADECIMENTOS}

Ao Conselho Nacional de Desenvolvimento Científico e Tecnológico (CNPq) - apoio financeiro: Edital Universal CNPq 15/2008-9 processo 476370/2007-8. 


\section{ABSTRACT}

Purpose: to identify the symptoms of changes in the exhalation of asthmatic children. Method: this study was conducted with 30 children from 6 to 10 year-old, of both genders and with asthma clinical diagnosis confirmed by medical records and 30 non-asthmatic children from 6 to 10 year-old, of both genders, and with same age. We evaluated the nasal ventilation with Altmann millimeter nasal mirror measuring the nasal air escape in order to check their unilateral or bilateral air output and the relation of symmetry between the right and left nostril. The images were imported into the computer through HP scanner - Scanjet 2400 series. The analysis was performed by the Scion Image software for Windows (Alpha 4.0.3.2). All conclusions were based on a significance level of 5\%, using Excel 2000 and SPSS v8.0 for the analysis. Results: there were no significant differences between asthmatic and non-asthmatic children about the measurements of total area and the right and left nostril areas. Conclusion: there were no symptoms of changes in the exhalation of asthmatic children, being necessary a more specific study as for the nasal and pulmonary functions.

KEYWORDS: Asthma; Child; Nasal Obstruction; Exhalation

\section{REFERÊNCIAS}

1. Bethlem N. Pneumologia. 4 ed. São Paulo: Atheneu, 2000.

2. Silva JRL, Nascentes R, Campos HS, Martire T. Asma brônquica. In: Aidé MA, Cardoso AP, Rufino R, David F, Carvalho SRS, Lucas VS et al. SOPTERJ/ Pneumologia: aspectos práticos e atuais. Rio de Janeiro: Revinter, 2001. p.201-10.

3. Birney MH, Brady CL, Bruchak KT, Carrillo C, Clark SH, Conley YP. Guia profissional para fisiopatologia. Rio de Janeiro: Guanabara Koogan, 2003.

4. Wannmacher L. Tratamento medicamentoso da asma em crianças. Uso racional de medicamentos: temas selecionados ISSN 1810-0791. 2006;3(9): 1-6.

5. II Diretrizes brasileiras no manejo da tosse crônica. J. bras. pneumol. J. bras. pneumol. 2006; 32(6): 403-s46.

6. Taketomi EA, Marra SMG, Segundo GRS. Fisioterapia em asma: efeito na função pulmonar e em parâmetros imunológicos. Fit Perform J. 2005;4(2):97-100.

7. Sousa DOC. O sistema estomatognático no respirador oral: fundamentos básicos para um diagnóstico precoce [monografia]. Fortaleza (CE): CEFAC - Saúde e Educação, 1999.

8.Tomé MC, Marchiori SC. Estudo eletromiográfico dos músculos orbiculares superior e inferior da boca em crianças respiradoras nasais e bucais durante $o$ repouso com e sem contato labial. J Bras Ortodon Ortop Facial. 1998;3(15):59-66.

9. Melo FMG, Cunha DA, Silva HJ. Avaliação da aeração nasal pré e pós a realização de manobras de massagem e limpeza nasal. Rev CEFAC. 2007;9(3):375-82.

10. Marchesan QI. Avaliação e terapia dos problemas da respiração. In: Marchesan QI. Fundamentos em fonoaudiologia: aspectos clínicos da motricidade oral. Rio de Janeiro: Guanabara Koogan; 1998. p.23-36.

11. Simon MS, Granato L, Oliveira RBC, Alcântara MPA. Rinoscleroma: relato de caso. Rev Bras Otorrinolaringol. 2006;72(4):568-71.

12. Calliari DS, Brescovici S, Kruse G. O espelho de Glatzel na avaliação da permeabilidade nasal antes e após exercício físico em indivíduos atletas. Rev Soc Bras Fonoaudiol. 2009;14(3):367-71.

13. Bucca C. Are asthma-like symptoms due to bronchiolar extra-thoracic airway dysfunction? Lancet. 1995;346(8978):786-7.

14. Ribeiro JD, Toro AADC, Baracat ECE. Antileukotrienes in the treatment of asthma and allergic rhinitis. J. Pediatr. (Rio J.). 2006; 82(5): 213-21.

15. Degan VV, Puppin-Rontani RMP. Aumento da aeração nasal após remoção de hábitos de sucção e terapia miofuncional. Rev CEFAC. 2007;9(1):55-60.

16. Branco A, Ferrari GF, Weber SA. Alterações orofaciais em doenças alérgicas de vias aéreas. Rev paul pediatr. 2007;25(3):266-70.

17. Cunha DA, Silva GAP, Motta MEFA, Silva HJ. A respiração oral em crianças e suas repercussões no estado nutricional. Rev CEFAC. 2007;9(1):47-54.

18. Gina - GLOBAL INITIATIVE FOR ASTHMA. Pocket guide for asthma management and prevention in children. 2006. [Disponível em: http:// www.ginasthma.com]. [Acesso em: 11 Set 2009]. 
19. IV Diretrizes Brasileiras para o manejo da asma. J. Bras Pneumol. 2006;32(7):447-74.

20. Fontes MJF, Fonseca MTM, Camargos AM, Affonso AGA, Calazaes GMC. Asmas em menores de cinco anos: dificuldades no diagnóstico e na prescrição de corticoterapia inalatória. J Bras Pneumol. 2005;31(3):244-53.

21. Penido FA, Noronha RMS, Caetano KI, Jesus MSV, Ninno CQMS, Britto ATBO. Correlação entre os achados do teste de emissão de ar nasal e da nasofaringoscopia em pacientes com fissura labiopalatina operada. Rev Soc Bras Fonoaudiol. 2007;12(2):126-34.

22. Bassi IB, Franco LP, Motta AR. Eficácia do emprego do espelho de Glatzel na avaliação da permeabilidade nasal. Rev. Soc. Bras. Fonoaudiol. 2009;14(3):367-71.
23.Di Francesco RC. Avaliação otorrinolaringológica da respiração oral. In: Krakauer LH, Di Francesco RC, Marchesan IQ. Respiração oral: abordagem interdisciplinar. São José dos Campos: Pulso; 2003. p.43-5.

24. Vidotti BA, Trindade IEK. Os efeitos da expansão rápida da maxila sobre a permeabilidade nasal avaliados por rinomanometria e rinometria acústica. Rev. Dent. Press Ortodon. Ortop. Facial. 2008 ; 13(6): 59-65

25. Yi LC, Jardim JR, Inoue DP, Pignatari SSN. Relação entre a excursão do músculo diafragma e as curvaturas da coluna vertebral em crianças respiradoras bucais. J. Pediatr. (Rio J.). 2008; 84(2): 171-7.

http://dx.doi.org/10.1590/S1516-18462011005000022

RECEBIDO EM: 11/02/2010

ACEITO EM: 18/10/2010

Endereço para correspondência:

Daniele Andrade da Cunha

Rua São Salvador, no 105 - Edifício São Salvador,

Apto 1002 - Espinheiro

Recife - PE

CEP: $52020-200$

E-mails: hdfono@yahoo.com.br dhahyfono@hotmail.com 